\title{
Adenine Phosphoribosyltransferase Deficiency: An Under-Recognized Cause of Urolithiasis and Renal Failure
}

\author{
Irène Ceballos-Picot ${ }^{1 *}$, Morgan Ledroit ${ }^{1}$, Lionel Mockel ${ }^{1}$, Véronique Droin ${ }^{1}$, Michel Daudon $^{2}$, Mohamad Zaidin $^{3}$, Jérôme Harambat ${ }^{4}$ and \\ Guillaume Bollée ${ }^{5}$
}

${ }^{1}$ Paris Descartes University, Sorbonne Paris Cité; Assistance Publique-Hôpitaux de Paris, Laboratory of Metabolomic and Proteomic Biochemistry; Center of reference Inherited metabolic diseases, Necker Universitary Hospital, Paris, France

${ }^{2}$ Functional Exploratory Unit, Assistance Publique-Hôpitaux de Paris, Tenon Hospital, Paris, France

${ }^{3}$ Department of Nephrology-Transplantation, Assistance Publique-Hôpitaux de Paris Necker Universitary Hospital, Paris, France and INSERM, U845, Paris, France

${ }^{4}$ Department of Pediatric Nephrology, Bordeaux Universitary Hospital, France

${ }^{5}$ Division of Nephrology and Research Center of the Centre Hospitalier de l'Université de Montréal and Université de Montréal, Québec, Canada

\begin{abstract}
Early diagnosis of monogenic forms of urolithiasis is important to prevent associated renal injury and other treatable disease manifestations, but is often delayed due to lack of knowledge of these rare disorders. Adenine phosphoribosyltransferase (APRT) deficiency is an under-recognized autosomal recessive disorder causing 2,8 dihydroxyadenine (2,8-DHA) urolithiasis and crystalline nephropathy secondary to intratubular 2,8-DHA crystalline precipitation. Patients often present with kidney stones but may also present with renal failure in the absence of stones or nephrocalcinosis. The disease can be efficiently treated by inhibitors of xanthine dehydrogenease (XDH), which makes early diagnosis and treatment essential to prevent recurrence of urolithiasis and nephropathy.
\end{abstract}

Here, we reviewed 67 patients from 56 families with complete APRT deficiency identified at Necker Universitary Hospital, Paris, France, between 1978 and 2014. The initial clinical presentation was urolithiasis in all symptomatic pediatric patients. In adult patients, recurrent nephrolithiasis was a much more common presentation (73\%) than crystalline nephropathy $(27 \%)$. Unfortunately, APRT deficiency was often misdiagnosed and irreversible loss of renal function occurred in $20 \%$ of cases.

APRT gene sequencing was performed in 54 patients (81\%) in 46 families: 25 pediatric patients in 22 families and 29 adult patients in 26 families. 98 mutated alleles were found out of 108 analyzed (91\%). Twenty seven different mutations were identified. A single $T$ insertion at the intron 4 splice donor site (c.400+2dup) leading to a truncated protein, accounted for 36 percent of mutated alleles for pediatric and adult cases.

This review summarizes the genetic data of a large cohort of pediatric and adult patients along with the issues for diagnosis and management of APRT deficiency and highlights the underdiagnosis associated with the potential severity of APRT deficiency. Early diagnosis is essential for treatment initiation and prevention of renal complications.

Keywords: Adenine phosphoribosyltransferase; APRT; Deficiency; Crystalline nephropathy; Urolithiasis; Renal failure; Prevention

\section{Introduction}

Adenine phosphoribosyltransferase (APRT) deficiency is an autosomal recessive disorder manifesting as urolithiasis or crystalline nephropathy $[1,2]$. It leads to the generation of insoluble 2,8-dihydroxyadenine (2,8-DHA) in urine promoting the formation of crystals and subsequently stones and/or precipitation in renal parenchyma inducing crystalline nephropathy. APRT enzyme provides the only pathway for the metabolic salvage of adenine and catalyzes the reaction between adenine and phosphoribosyl pyrophosphate (PRPP) to form 5'-adenosine monophosphate (AMP) (Figure 1). In APRT defect, adenine is oxidized into 2,8-DHA by xanthine dehydrogenase (XDH). 2,8-DHA has a high renal clearance, which may involve both filtration and tubular secretion [3]. The polyamine pathway has been identified as the main source of endogenous adenine in humans [4] and adenine-rich foods may be a contributing factor in precipitating urolithiasis. APRT deficiency thereby results in excretion of large amounts of 2,8-DHA in urine. 2,8-DHA is extremely insoluble in urine and forms crystals, which can aggregate into stones or precipitate into tubular lumens, inside renal epithelial cells, and in the interstitium, resulting in crystalline 2,8-DHA nephropathy [1,2,4-6].

The kidneys and urinary tract system seem to be the only organs affected by APRT deficiency [7]. Recent reports highlight the risk of renal allograft loss in patients with undiagnosed APRT deficiency [811 ] and the need for improved early detection of the disease. Early diagnosis of APRT deficiency is critical because patients may develop chronic kidney disease (CKD) and in some case irreversible kidney failure that may have been efficiently prevented by allopurinol, a $\mathrm{XDH}$ inhibitor (Figure 1). Tools for diagnosis include stone analysis, identification of typical 2,8-DHA crystals in urine or renal biopsy, and measurement of APRT activity in erythrocyte lysates.

Two different types of APRT deficiency have been described with similar clinical expression and complete APRT deficiency in vivo but have been distinguished on the level of enzyme activity in cell extracts in vitro. Type I (complete deficiency in vivo and in vitro) has been reported in different ethnic groups and predominantly affects Caucasian population $[1,2,5,9,12]$. By contrast, type II (complete deficiency in vivo but partial deficiency in vitro) has been observed almost exclusively in the Japanese population [13].

*Corresponding author: Irène Ceballos-Picot, Laboratory of Metabolomic and Proteomic Biochemistry, Necker Universitary Hospital, Paris, France, Tel: 0033144495361; Fax: 0033144495130; E-mail: irene.ceballos@nck.aphp.fr

Received April 25, 2014; Accepted June 28, 2014; Published July 04, 2014

Citation: Picot IC, Ledroit M, Mockel L, Droin V, Daudon M, et al. (2014) Adenine Phosphoribosyltransferase Deficiency: An Under-Recognized Cause of Urolithiasis and Renal Failure. J Nephrol Ther 4: 173. doi:10.4172/2161-0959.1000173

Copyright: ( 2014 Picot IC, et al. This is an open-access article distributed under the terms of the Creative Commons Attribution License, which permits unrestricted use, distribution, and reproduction in any medium, provided the original author and source are credited. 


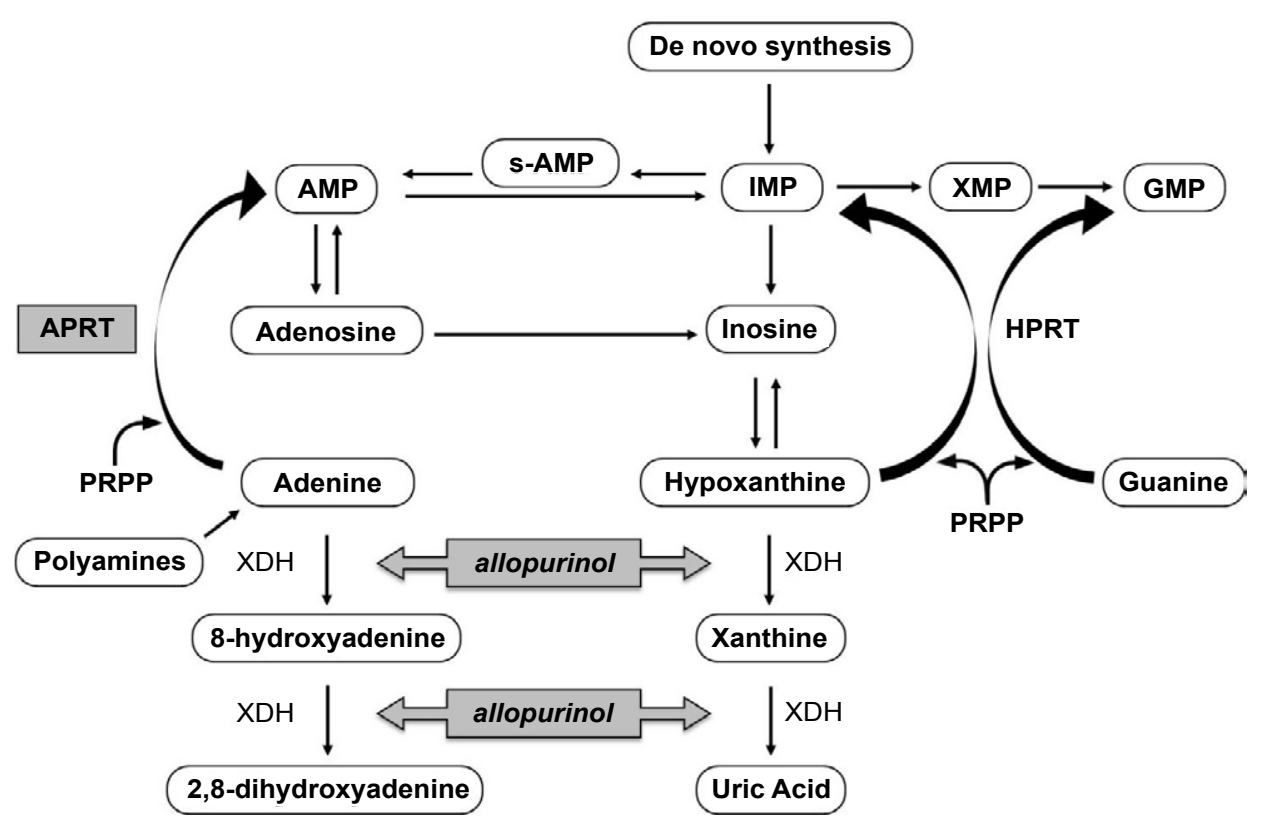

Figure 1: APRT deficiency causes 2,8-DHA formation. In humans, adenine cannot be converted to adenosine as hypoxanthine to inosine; the only alternative pathway in APRT deficiency is oxidation of adenine to 2,8-DHA by xanthine dehydrogenase (XDH) via the 8-hydroxy-intermediate. Allopurinol acts by inhibiting XDH, thus preventing 2,8-DHA synthesis. AMP: adenosine monophosphate, GMP: guanosine monophosphate, HPRT: hypoxanthine-guanine phosphoribosyltransferase, IMP: inosine monophosphate, PRPP: 5-phosphoribosyl-1-pyrophosphate.

The aprt gene located on chromosome $16 \mathrm{q} 24$ is about $2.6 \mathrm{~kb}$ long and contains 5 exons [14]. Mutant alleles responsible for the disease have been classified as $\mathrm{APRT}^{\star} \mathrm{Q} 0$ for type I and APRT ${ }^{\star} \mathrm{J}$ for type II. $\mathrm{APRT}^{\star} \mathrm{Q} 0$ represents a heterogeneous collection of mutations $[1,4,15$ 17] and patients with type I deficiency are either homozygotes or compound heterozygotes for these mutations. APRT ${ }^{\star} \mathrm{J}$ is a single mutant allele with a missense mutation in exon 5 (M136T) [18] and patients with type II deficiency have the genotype APRT ${ }^{\star} \mathrm{J} / \mathrm{APRT}^{\star} \mathrm{J}$ or more rarely $\mathrm{APRT}^{\star} \mathrm{J} / \mathrm{APRT}^{\star} \mathrm{Q} 0[19]$.

Cases of APRT deficiency were only exceptionally reported in occidental countries. Most cases have been reported from Iceland and France $[1,2,5,7]$. Disease incidence in Iceland is high. A founder effect is suspected, based on reports of common mutations in Iceland [5]. More than twenty years ago, we drew attention to the under diagnosis of APRT deficiency in Caucasian population, considering the surprisingly small number of cases reported despite a homozygosity at APRT locus estimated at 1 in 50.000 to 1 in 100.000 [20]. The most plausible explanation was that APRT deficiency may be largely unrecognized due to lack of knowledge of the disease, inadequate evaluation of kidney stone, and confusions of 2,8-DHA crystals or calculi with uric acid or calcium oxalate.

Our report reviews the genetic basis of APRT deficiency in a large cohort of APRT-deficient patients, highlights the potential severity of the disease and the crucial importance of early recognition to initiate treatment and prevent renal complications. Diagnostic tools helpful for a better diagnosis of APRT deficiency are also reviewed.

\section{Clinical Presentation at Diagnosis of APRT Deficiency}

We retrospectively reviewed all cases of APRT deficiency identified between 1978 and 2014 in the Biochemistry laboratories (Necker Universitary Hospital, Paris, France) which are referral centers for urolithiasis and diagnosis of inborn errors of purine metabolism. Most patients were not followed at Necker Hospital but blood and/ or urine samples were sent from other centers. Diagnosis of complete APRT deficiency was determined by null APRT enzymatic activity in erythrocytes (sex ratio M/F: 39/28). APRT deficiency was found in 67 patients from 56 families: 27 pediatric cases (age of first symptoms $<16$ years) and 40 adult cases (age of first symptoms $>16$ years), at a median age of 31.3 years (range $0.5-73$ ). The median age at diagnosis for adult cases was 46 years (range 22 to 73 ), and 4.5 years for pediatric cases (range 0.5 to 15 ).

A striking finding was a diagnosis delay for years from the beginning of symptoms in $70 \%$ of patients. Diagnosis of APRT deficiency was based on one or several of the following items: 1) identification of typical 2,8-DHA crystals in urine, 2) 2,8-DHA in stone analysis, 3) null APRT activity in erythrocyte lysates, 4) identification of 2,8-DHA crystals in renal biopsy. When available, patient's medical charts were reviewed in order to collect data, such as age when the first clinical manifestation occurred as well as age and clinical presentation at diagnosis. Episodes of urolithiasis were defined as one of the following: 1) spontaneous stone expulsion, 2) renal colic, 3) radiological detection of a new stone. Renal function was estimated using MDRD (Modification of Diet in Renal Disease Study) formula.

The initial clinical presentation was urolithiasis in 24 of the 27 symptomatic pediatric cases (89\%), one infant exhibited nephrocalcinosis. Two children were diagnosed by familial screening and were asymptomatic at time of diagnosis. Age at diagnosis was before 3 years of life in 10 children, between 4 and 10 years of age in 9 children and between 11 and 16 years of age for 2 children (Figure 2). Diagnosis was made within 3 months after the first episode of lithiasis in $75 \%$ of children. A considerable delay in diagnosis occurred in $25 \%$ of children. In these children, diagnosis was established after a median time of 2 years and after a median number of four stone passage episodes [21].

The initial clinical presentation was urolithiasis for $73 \%$ of adult 
Citation: Picot IC, Ledroit M, Mockel L, Droin V, Daudon M, et al. (2014) Adenine Phosphoribosyltransferase Deficiency: An Under-Recognized Cause of Urolithiasis and Renal Failure. J Nephrol Ther 4: 173. doi:10.4172/2161-0959.1000173

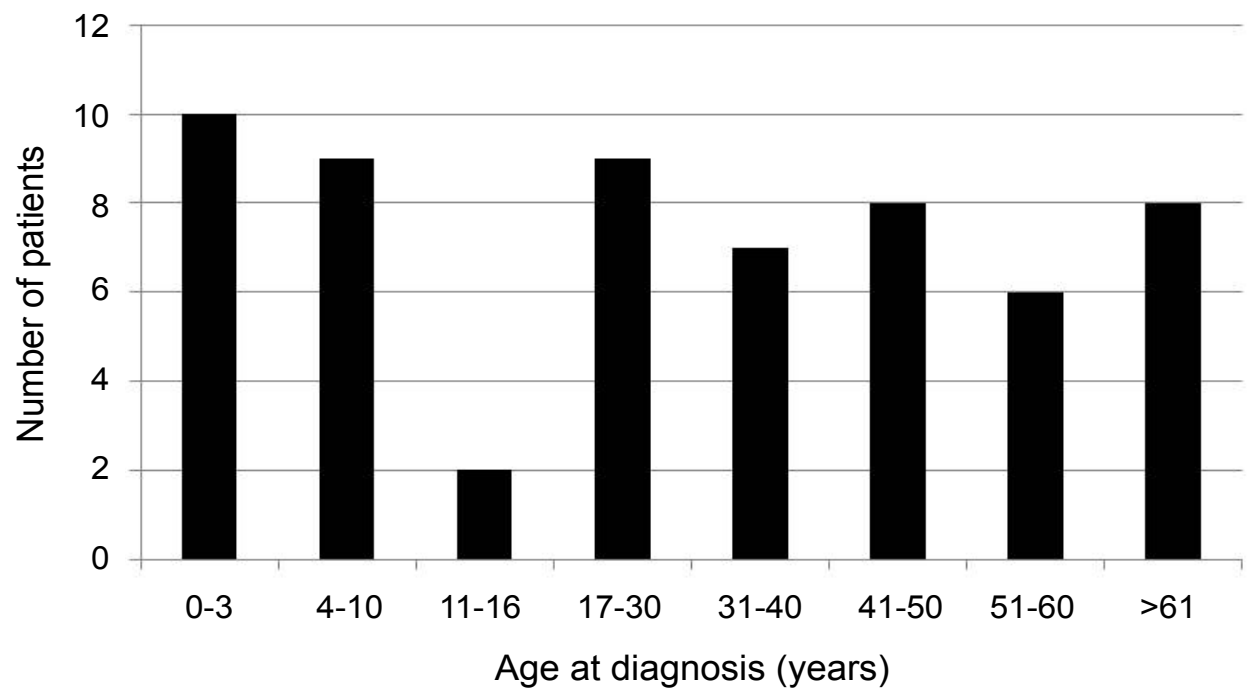

Figure 2: Age at diagnosis (years). Distribution of patients according to age at diagnosis of APRT deficiency.

\begin{tabular}{|c|c|}
\hline Characteristic & Value \\
\hline Number of families & 56 \\
\hline Number of patients & 67 \\
\hline Pediatric cases $<16$ years $[n(\%)]$ & $27(40)$ \\
\hline Adult cases >16 years [n (\%)] & $40(60)$ \\
\hline Male gender [n (\%)] & $39(58)$ \\
\hline Female gender [n (\%)] & $28(42)$ \\
\hline Age at diagnosis [years (median)] & $31.3(0.5$ to 73$)$ \\
\hline Age at diagnosis pediatric cases [years (range)] $(n=21)$ & $4.5(0.5$ to 15$)$ \\
\hline Age at diagnosis adult cases [years (range)] $(n=38)$ & $46(22$ to 73$)$ \\
\hline Number of patients with age information about first symptoms [n (\%)] & $27(40)$ \\
\hline Number of patients with diagnosis delay [n (\%)] & $19(70)$ \\
\hline Diagnosis delay $0-10$ years [n (\%)] & $9(47)$ \\
\hline Diagnosis delay $10-43$ years [n (\%)] & $10(53)$ \\
\hline Presymptomatic diagnosis [n (\%)] & $2(3)$ \\
\hline \multicolumn{2}{|l|}{ Clinical presentation at diagnosis } \\
\hline \multicolumn{2}{|l|}{ Lithiasis [n (\%)] } \\
\hline Pediatric cases & $25(93)$ \\
\hline Adult cases & $29(73)$ \\
\hline \multicolumn{2}{|l|}{ Crystalline nephropathy [n (\%)] } \\
\hline Pediatric cases & $0(0)$ \\
\hline Adult cases & $11(27)$ \\
\hline \multicolumn{2}{|l|}{ Renal graft [n (\%)] } \\
\hline Pediatric cases & $0(0)$ \\
\hline Adult cases ( 5 crystalline nephropathy) & $8(20)$ \\
\hline
\end{tabular}

Table 1: Characteristics of patients identified with APRT deficiency.

cases (29/40 patients) and crystalline nephropathy for 27\% (11/40 adult patients) with a progression to end-stage renal disease (ESRD) leading to renal graft for $20 \%$ (8/40 adult patients) (Table 1$)$. Recurrent nephrolithiasis is a much more common presentation than crystalline nephropathy. Figure 2 shows the repartition of adult patients depending of their age at diagnosis. A striking finding was a diagnosis delay in $70 \%$ of patients. Delay from first episode of urolithiasis to diagnosis was extremely variable ranging from 0 to 43 years. Eight patients reached ESRD and underwent transplantation before the diagnosis was made, APRT deficiency was diagnosed in the setting of severe allograft dysfunction caused by intratubular and interstitial precipitation of 2,8DHA crystals.
APRT deficiency varies in presentation and natural history. Since patients are treated with allopurinol after diagnosis it is difficult to determine what proportion of patients would progress to ESRD if untreated? In our French cohort, among patients who were diagnosed and treated later than 40 years of age, $43 \%$ of patients had eGFR $>60$ $\mathrm{ml} / \mathrm{min} / 1.73 \mathrm{~m}^{2}, 22 \%$ had eGFR of 30 to $60 \mathrm{ml} / \mathrm{min} / 1.73 \mathrm{~m}^{2}$ and $35 \%$ had eGFR $<15 \mathrm{ml} / \mathrm{min} / 1.73 \mathrm{~m}^{2}$. at diagnosis.

The age at diagnosis of APRT deficiency varies widely, ranging from infancy to more than 70 years. In our series, diagnosis was made before the age of 16 years in $40 \%$ of patients. In an Icelandic series, $47 \%$ of affected individuals were diagnosed before age of 18 years $[1,12,13]$. In some instances, diagnosis of APRT deficiency was 
delayed (sometimes by decades) either because patients had remained remarkably asymptomatic or there was insufficient clinical suspicion despite early onset of recurrent urolithiasis and chronic kidney disease $[1,2]$. Urolithiasis is the most common clinical manifestation of APRT deficiency in both children and adults [1,5,7,21,22]. The first urolithiasis episode may occur during infancy as well as later than the age of 40. In affected infants, reddish-brown diaper stains related to 2,8-DHA crystalluria can be observed [1]. In some instances, bilateral 2,8-DHA stones can cause urinary tract obstruction and acute renal failure, especially in children [21,23,24]. 2,8-DHA stones are usually radiolucent and thus can be detected only by imaging techniques capable of detecting radiolucent stones, such as ultrasound or computed tomography. However, 2,8-DHA stones may sometimes appear as radiopaque when containing calcium salts [25]. Due to their radiolucent character, 2,8-DHA stones are often mistaken for uric acid stones.

2,8-DHA crystalline nephropathy represents the second manifestation of APRT deficiency. In our series, 2,8-DHA crystalline nephropathy was observed in $27 \%$ of adults but not in children. Crystalline nephropathy occurs in patients who have remained undiagnosed and untreated despite a history of recurrent urolithiasis. However, it should be emphasized that crystalline nephropathy can occur in patients who experienced only a few stone episodes or exceptionally in patients with no history of urolithiasis [6]. Imaging studies demonstrating absence of stone do not definitively rule out the possibility of 2,8-DHA nephropathy. 2,8-DHA nephropathy usually develops insidiously and cause chronic kidney disease progressing over a period of years. Less commonly, 2,8-DHA nephropathy can present as an acute or subacute disease. Massive precipitation of 2,8-DHA into the kidney can sometimes be triggered by urine concentration and saturation of 2,8-DHA in the context of dehydration. In both European and Japanese cohorts, decreased renal function was observed in about one-third of patients at time of diagnosis and nearly $10 \%$ of patients had reached ESRD prior to diagnosis $[1,2,5,7,12,13]$.

For 8 patients in our cohort ( $20 \%$ of the adult cases) the diagnosis of APRT deficiency was made after renal transplantation. Several other cases of crystalline nephropathy recurring after renal transplantation and leading to irremediable loss of allograft function within a few weeks have been reported [6,8-10,26,27]. In most tragic cases, several renal transplantations failed before APRT deficiency was properly recognized and treated $[8,10]$.

Nearly 15 percent of individuals with APRT deficiency may be asymptomatic [1,5] but are at risk of developing pathological manisfestations if untreated. The factors underlying the variability of APRT deficiency phenotype are unclear. Environmental factors such as fluids and purine intake, acute episodic dehydratation, are likely to account for phenotypic variability. The potential influence of modifying genes has not been reported in humans. One study in mice suggested that individual differences in the effectiveness of crystallization inhibitors such as osteopontin may modulate the expression of the disease [28].

APRT deficiency is not known to cause extrarenal manifestations although clinical observations in some heterozygous patients suggested that partial APRT deficiency could contribute to hyperuricemia and gout $[29,30]$. This was the case for 3 heterozygous patients among 59 with partial APRT deficiency (5\%). Heterozygotes showed absence of 2,8-DHA crystalluria, despite partial APRT deficiency. APRT activity from erythrocyte lysates was studied in 59 heterozygotes diagnosed during the screening in 26 families: $73 \%$ of $\mathrm{APRT}^{\star} \mathrm{Q} 0$ heterozygotes (43/59) exhibited $20-30 \%$ residual APRT activity and $27 \%$ of heterozygotes (16/59) showed only $10-15 \%$ residual activity. Crystalluria was negative for this last group and all heterozygotes were asymptomatic. However, one case of 2,8-DHA urolithiasis was reported in a patient carrying one mutant $\mathrm{APRT}^{\star} \mathrm{J}$ and one normal allele [31]. No other case of symptomatic heterozygote has been reported to date.

In summary, diagnosis was delayed in many patients despite the presence of symptoms for many years. $20 \%$ of our cohort developed ESRD and diagnosis of APRT deficiency was only made post renal transplant in the setting of allograft dysfunction (severe and irreversible in some cases). Thus, this condition remains poorly recognized despite the availability of diagnostic tools.

\section{Molecular Genetic Study in APRT-deficient Pediatric and Adult Cases and Prevalence}

Approximately 90 families with type I defect, predominantly Caucasians, have been reported from many different countries, including over 30 from Japan $[1,2]$. The type II deficiency has been identified in more than 70 other Japanese families [4,32]. Various mutations reported include missense $[15,18,33,34]$, non-sense $[33,35]$, insertion or deletion $[17,19,33]$ and mutation at the splice junction site leading to abnormal mRNA splicing $[17,32,33,36]$.

The five exons and flanking regions of aprt gene were amplified by PCR and then sequenced as described in [1] and [32] in 54 patients belonging to 46 families: 25 pediatric patients in 22 families and 29 adult patients in 26 families. 98 mutated alleles were found on the 108 analyzed (91\%). Twenty seven different mutations were identified. The distribution of the mutations throughout the gene is indicated in Figure 3 including the number of mutated alleles for each mutation. Among the 27 different mutations: 14 were specifically found in adult cases, 9 specific to pediatric patients and only 4 mutations were common to adult and pediatric patients including c.400+2dup (Figure 3).

A single $\mathrm{T}$ insertion at the intron 4 splice donor site (c.400+2dup) leading to a truncated protein (Figure 4), accounted for 36 percent of mutated alleles for pediatric and adult cases. This c.400+2dup mutation occurred on the aprt allele carrying the polymorphic TaqI site, suggesting a founder effect in some families [37]. In the Caucasian population there are two common aprt mutations that show uniform associations with highly polymorphic restriction sites for TaqI and SphI [38], namely a missense mutation in British and Icelandic patients [15,37], and c.400+2dup, which seems to be the most common cause of APRT deficiency among Caucasians [1,2,17,33,37]. Of note, c. $400+2$ dup mutation was only detected in families from metropolitan France and one Italian family. Families from other countries carried other mutations at homozygous state, suggesting consanguinity or uniparental disomy of chromosome 16 [32].

Mutations at the cDNA level and their expected effect on protein are summarized in Table 2 for pediatric patients and Table 3 for adult patients. Of these, 25 patients were carrying a homozygous mutation and 27 had compound heterozygous mutations. The most prevalent mutation c. $400+2$ dup is at the homozygous state for 10 patients ( 7 families) and at the heterozygous state for 19 patients (16 families): 54 percent of patients (50 percent of families) harbour the c.400+2dup mutation at the heterozygous or homozygous state. Aberrant splicing at this site causes deletion of exon 4 leading to a frameshift and a truncated protein (Ala108Glufs ${ }^{\star} 3$ ) of 109 amino acids instead of 180 (Figure 4). We evaluated the prevalence of c.400+2dup mutant allele in the general population by performing a molecular study in 102 


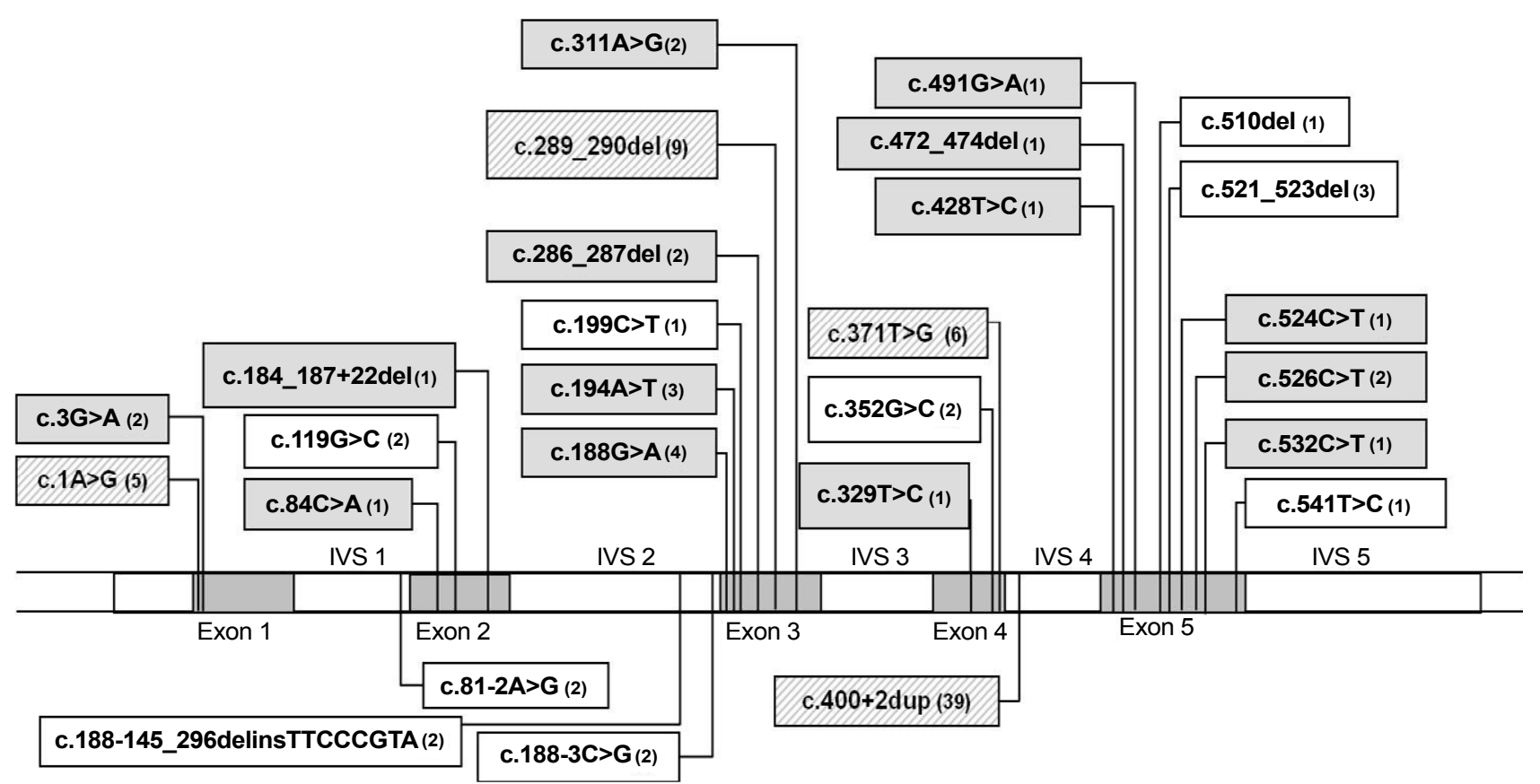

Figure 3: Distribution of causal mutations throughout the aprt gene. White box: mutations only found in pediatric patients (< 16 years). Grey box: mutations only found in adult patients (> 16 years). Hachured box: mutations common to pediatric and adult patients. In brackets: number of mutated alleles for each mutation.

\section{A Normal genomic DNA}

c. $400+2$ dup mutation

\section{5' ACTGGTG:GTAAGGGTC 3' \\ 1830}

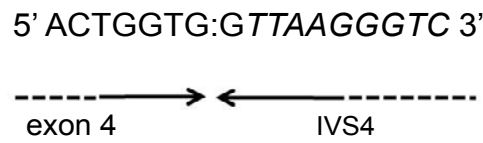

B

Normal

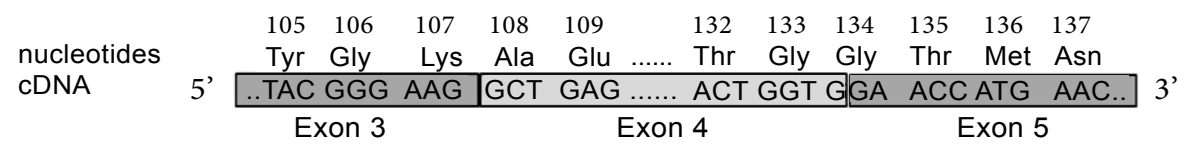

c. $400+2$ dup

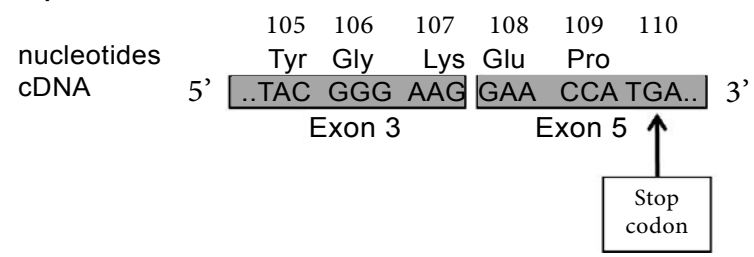

Figure 4: Nucleotide sequence at the exon 4-intron 4 junction showing the most prevalent mutation c.400+2dup. T insertion between nucleotides 1831 - 1832 or 1832 1833 at the IVS4 splice donor site (A) results in deletion of exon 4 in mRNA, leading to a premature termination at amino acid 110 (Ala108Glufs 3 ) (B) (adapted from 1 and 17).

healthy newborns: c.400+2dup mutation was found in 2 out of 204 chromosomes examined (1\%).

The c.400+2dup mutation had been identified previously in several families from Europe and seems to be the most common cause of APRT deficiency in this population [1,2,15,17,37,38]. Another common mutation is a missense (Asp65Val) found in British, Icelandic and Spanish patients $[1,15,37]$. Asp65Val mutation was found in the homozygous state in all of the sixteen families reported in an Icelandic study [5], which suggested a founder effect. 


\begin{tabular}{|c|c|c|c|c|c|c|c|}
\hline \multicolumn{2}{|c|}{ Pediatric cases } & \multicolumn{3}{|c|}{$1^{\text {st }}$ Allele } & \multicolumn{3}{|c|}{$2^{\text {nd }}$ Allele } \\
\hline Patients & Gender & Region & cDNA & Protein & Region & cDNA & Protein \\
\hline 2 * & M & Intron 4 & c.400+2dup & p.Ala108Glufs*3 & Intron 4 & c. $400+2$ dup & p.Ala108Glufs*3 \\
\hline $3 *$ & M & Intron 4 & c. $400+2$ dup & p.Ala108Glufs*3 & Intron 4 & c. $400+2$ dup & p.Ala108Glufs*3 \\
\hline $4 \#$ & M & Intron 4 & c. $400+2$ dup & p.Ala108Glufs*3 & Exon 5 & c. $526 \mathrm{C}>\mathrm{T}$ & p.Leu176Phe \\
\hline $5 \#$ & $M$ & Intron 4 & c. $400+2$ dup & p.Ala108Glufs ${ }^{*} 3$ & Exon 5 & c. $526 \mathrm{C}>\mathrm{T}$ & p.Leu176Phe \\
\hline 6 & M & Exon 1 & c. $3 G>A$ & p.Met1? & Exon 1 & c. $3 \mathrm{G}>\mathrm{A}$ & p.Met1? \\
\hline 8 & $M$ & Exon 3 & c.289_290del & p.Leu97Valfs*12 & Intron 4 & c. $400+2$ dup & p.Ala108Glufs*3 \\
\hline 13 & $M$ & Exon 3 & c. $194 \mathrm{~A}>\mathrm{T}$ & p.Asp65Val & Exon 4 & c. $329 \mathrm{~T}>\mathrm{C}$ & p.Leu110Pro \\
\hline 14 & M & Intron 4 & c. $400+2$ dup & p.Ala108Glufs*3 & NF & NF & NF \\
\hline 16 & $\mathrm{~F}$ & Intron 4 & c. $400+2$ dup & p.Ala108Glufs*3 & Intron 4 & c. $400+2$ dup & p.Ala108Glufs*3 \\
\hline 21 & $\mathrm{~F}$ & Exon 4 & c. $371 \mathrm{~T}>\mathrm{G}$ & p.Val124Gly & Exon 4 & c. $371 \mathrm{~T}>\mathrm{G}$ & p.Val124Gly \\
\hline 26 & M & Exon 5 & c. $532 \mathrm{C}>\mathrm{T}$ & p.GIn178* & NF & NF & NF \\
\hline 28 & $\mathrm{~F}$ & Intron 4 & c. $400+2$ dup & p.Ala108Glufs*3 & Exon 5 & c. $524 \mathrm{C}>\mathrm{T}$ & p.Ser175Phe \\
\hline 35 & $\mathrm{~F}$ & Intron 4 & c. $400+2$ dup & p.Ala108Glufs*3 & Intron 4 & c. $400+2$ dup & p.Ala108Glufs ${ }^{*} 3$ \\
\hline $38^{\circ}$ & $M$ & Exon 3 & c.289_290del & p.Leu97Valfs*12 & Intron 4 & c. $400+2$ dup & p.Ala108Glufs*3 \\
\hline $39^{\circ}$ & M & Exon 3 & c.289_290del & p.Leu97Valfs ${ }^{*} 12$ & Intron 4 & c. $400+2$ dup & p.Ala108Glufs*3 \\
\hline 40 & $\mathrm{~F}$ & Intron 4 & c. $400+2$ dup & p.Ala108Glufs*3 & NF & NF & NF \\
\hline 46 & $M$ & Exon 3 & c. $188 \mathrm{G}>\mathrm{A}$ & p.Gly63Asp & Exon 3 & c. $188 \mathrm{G}>\mathrm{A}$ & p.Gly63Asp \\
\hline 48 & M & Exon 3 & c. $194 \mathrm{~A}>\mathrm{T}$ & p.Asp65Val & Exon 3 & c. $194 \mathrm{~A}>\mathrm{T}$ & p.Asp65Val \\
\hline 49 & $\mathrm{~F}$ & Exon 3 & c. $188 \mathrm{G}>\mathrm{A}$ & p.Gly63Asp & Exon 3 & c. $188 \mathrm{G}>\mathrm{A}$ & p.Gly63Asp \\
\hline 51 & M & Exon 3 & c.286_287del & p.Thr96Serfs*13 & Exon 3 & c.286_287del & p.Thr96Serfs*13 \\
\hline 52 & M & Exon 2 & c. $84 \mathrm{C}>\mathrm{A}$ & p.Asp28Glu & $\begin{array}{l}\text { Exon } 2 \text { I } \\
\text { Intron } 2\end{array}$ & c.184_187+22del & p.Gly63* \\
\hline 55 & $\mathrm{~F}$ & Intron 4 & c. $400+2$ dup & p.Ala108Glufs ${ }^{\star} 3$ & Exon 5 & c. $472 \_474 \mathrm{del}$ & p.Glu158del \\
\hline $58 \therefore$ & $\mathrm{F}$ & Exon 1 & c. $1 A>G$ & p.Met1? & Exon 5 & c. $491 \mathrm{G}>\mathrm{A}$ & p.Gly164Asp \\
\hline $59 \therefore$ & $\mathrm{F}$ & ND & ND & ND & ND & ND & ND \\
\hline $60 \therefore$ & $\mathrm{F}$ & ND & ND & ND & ND & ND & ND \\
\hline 65 & M & Intron 4 & c. $400+2$ dup & p.Ala108Glufs*3 & Exon 5 & c. $428 \mathrm{~T}>\mathrm{C}$ & p.Leu143Pro \\
\hline 66 & $M$ & Exon 3 & c. $311 \mathrm{~A}>\mathrm{G}$ & p.Glu104Gly & Exon 3 & c. $311 A>G$ & p.Glu104Gly \\
\hline
\end{tabular}

ND: not determined; NF: not found

One symbol for one family $\left({ }^{*}, \#,{ }^{\circ}, \therefore\right)$

Table 2: Aprt gene mutations in pediatric patients (<16 years)

Type II APRT deficiency is caused by a single mutant allele with a missense mutation in exon 5 (Met136Thr) referred as APRT ${ }^{\star}$, which has been reported exclusively in the Japanese population [13,17-19]. Type II is associated with genotype $\mathrm{APRT}^{\star} \mathrm{J} / \mathrm{APRT}^{\star} \mathrm{J}$ or more rarely $\mathrm{APRT}^{\star} \mathrm{J} / \mathrm{APRT}^{*} \mathrm{Q} 0[19]$. An exception to this is a missense mutation (V150F) reported in a patient of Polish origin, which produced an enzyme with null activity in vivo but residual activity in cell extracts [16]. Two-thirds of our families originated from metropolitan France, however some families originated from Martinique, Poland, Italy, Spain, Turkey, Lebanon, Canada and African countries [1,2], suggesting that APRT deficiency affects people worldwide.

APRT deficiency is often considered a very rare disease although its worldwide prevalence remains unknown. The vast majority of reported cases arise from Japan, France and Iceland $[1,2,5,7,13]$. This may be related to high frequency of specific mutations in these countries (Met136Thr in Japan, c.400+2dup in France and Asp65Val in Iceland). However, variability in awareness of APRT deficiency and availability of diagnostic tests among different countries are likely to account for the variability in number of cases identified. This suggests that APRT deficiency affects people worldwide. In France, the number of patients diagnosed with complete APRT deficiency has notably increased in the last few years, which probably reflects improved recognition and diagnosis of the disease [2]. The prevalence of complete APRT deficiency was estimated to about $1 / 27.000$ of the Japanese population, corresponding to a heterozygote frequency higher than $1.2 \%$ [13]. The heterozygote frequency in healthy caucasian populations, estimated from measurements of APRT enzyme activity, ranges from 0.4 to $1.2 \%$ $[39,40]$, suggesting that expected homozygosis should range from $1 / 50.000$ to $1 / 100.000[20,41]$. If this holds true, more than 60,000 80,000 individuals may be affected worldwide, of whom 40000 would be expected in Asia, 9000 in Europe and 8000 in America, including at least 3000 affected individuals in the USA [42]. This contrast with the small number of cases recorded in most countries and suggests that the majority of individuals with APRT deficiency are currently unrecognized [20]. APRT deficiency may be a seriously underestimated cause of urolithiasis and chronic kidney disease, progressing over time to ESRD in a non-negligible proportion of cases when left untreated.

No genotype-phenotype correlations have been established; clinical features are known to vary greatly between individuals harbouring the same mutations $[1,5]$.

\section{Early diagnosis of APRT deficiency is the key to prevention}

Clinical recognition of APRT deficiency can be challenging, due 
Page 7 of 11

\begin{tabular}{|c|c|c|c|c|c|c|c|}
\hline \multicolumn{2}{|c|}{ Adult cases } & \multicolumn{3}{|c|}{$1^{\text {st }}$ Allele } & \multicolumn{3}{|c|}{$2^{\text {nd }}$ Allele } \\
\hline Patients & Gender & Region & cDNA & Protein & Region & cDNA & Protein \\
\hline 1 & M & Intron 4 & c. $400+2$ dup & p.Ala108Glufs*3 & Intron 4 & c. $400+2$ dup & p.Ala108Glufs*3 \\
\hline 7 & M & ND & ND & ND & ND & ND & ND \\
\hline $9 \sim$ & M & ND & ND & ND & ND & ND & ND \\
\hline $10 \sim$ & $M$ & ND & ND & ND & ND & ND & ND \\
\hline 11 & M & Exon 3 & c.289_290del & p.Leu97Valfs*12 & Exon 5 & c.521_523del & p.Phe174del \\
\hline 12 & $M$ & Exon 1 & c. $1 A>G$ & p.Met1? & Intron 4 & c. $400+2$ dup & p.Ala108Glufs $* 3$ \\
\hline 15 & $M$ & ND & ND & ND & ND & ND & ND \\
\hline 17 & M & ND & ND & ND & ND & ND & ND \\
\hline 18 & $\mathrm{~F}$ & ND & ND & ND & ND & ND & ND \\
\hline 19 & $\mathrm{~F}$ & Intron 4 & c. $400+2$ dup & p.Ala108Glufs*3 & NF & $\mathrm{NF}$ & NF \\
\hline 20 & $\mathrm{~F}$ & Intron 4 & c. $400+2$ dup & p.Ala108Glufs*3 & Intron 4 & c. $400+2$ dup & p.Ala108Glufs $* 3$ \\
\hline $22 \cdot$ & $M$ & Exon 4 & c. $371 T>G$ & p.Val124Gly & Exon 4 & c. $371 T>G$ & p.Val124Gly \\
\hline 23 . & $\mathrm{F}$ & Exon 4 & c. $371 \mathrm{~T}>\mathrm{G}$ & p.Val124Gly & Exon 4 & c. $371 \mathrm{~T}>\mathrm{G}$ & p.Val124Gly \\
\hline 24 & $M$ & ND & ND & ND & ND & ND & ND \\
\hline 25 & $\mathrm{~F}$ & Intron 4 & c. $400+2$ dup & p.Ala108Glufs*3 & Intron 4 & c. $400+2$ dup & p.Ala108Glufs $* 3$ \\
\hline 27 & M & Intron 2 / Exon 3 & $\begin{array}{l}\text { c.188-145_296del } \\
\text { insTTCCCGTA }\end{array}$ & p.? & Intron 2 / Exon 3 & $\begin{array}{l}\text { c.188-145_296del } \\
\text { insTTCCCGTA }\end{array}$ & p.? \\
\hline 29 & M & Intron 2 & c. $188-3 C>G$ & p.? & Intron 2 & c. $188-3 C>G$ & p.? \\
\hline 30 & $\mathrm{~F}$ & Intron 1 & c. $81-2 A>G$ & p.? & Intron 1 & c. $81-2 A>G$ & p.? \\
\hline 31 & $\mathrm{~F}$ & Exon 3 & c.289_290del & p.Leu97Valfs ${ }^{*} 12$ & Exon 3 & c.289_290del & p.Leu97Valfs ${ }^{*} 12$ \\
\hline 32 & $\mathrm{~F}$ & Exon 3 & c. $199 C>T$ & p.Arg67* & Intron 4 & c. $400+2$ dup & p.Ala108Glufs*3 \\
\hline 33 & $\mathrm{~F}$ & Intron 4 & c. $400+2$ dup & p.Ala108Glufs*3 & Exon 5 & c.521_523del & p.Phe174del \\
\hline 34 & M & Intron 4 & c. $400+2$ dup & p.Ala108Glufs*3 & NF & NF & $\mathrm{NF}$ \\
\hline 36 & $M$ & Intron 4 & c. $400+2$ dup & p.Ala108Glufs*3 & Exon 5 & c.510del & p.Val171Tyrfs*82 \\
\hline 37 & M & Exon 3 & c.289_290del & p.Leu97Valfs ${ }^{\star 12}$ & Intron 4 & c.400+2dup & p.Ala108Glufs*3 \\
\hline 41 & $\mathrm{~F}$ & Exon 5 & c. $541 \mathrm{~T}>\mathrm{C}$ & p. ${ }^{*} 181$ Argext $^{\star} 121$ & NF & NF & NF \\
\hline 42 & $\mathrm{~F}$ & ND & ND & ND & ND & ND & ND \\
\hline 43 & $\mathrm{~F}$ & Intron 4 & c. $400+2$ dup & p.Ala108Glufs*3 & Intron 4 & c. $400+2$ dup & p.Ala108Glufs*3 \\
\hline 44 & M & Intron 4 & c. $400+2$ dup & p.Ala108Glufs*3 & Intron 4 & c. $400+2$ dup & p.Ala108Glufs $* 3$ \\
\hline 45 & $\mathrm{~F}$ & Intron 4 & c. $400+2$ dup & p.Ala108Glufs*3 & Intron 4 & c. $400+2$ dup & p.Ala108Glufs $* 3$ \\
\hline 47 & $\mathrm{~F}$ & NF & NF & NF & NF & NF & NF \\
\hline 50 & $\mathrm{~F}$ & Exon 3 & c.289_290del & p.Leu97Valfs ${ }^{*} 12$ & Exon 3 & c.289_290del & p.Leu97Valfs ${ }^{*} 12$ \\
\hline 53 & $M$ & Exon 1 & c. $1 A>G$ & p.Met1? & Intron 4 & c. $400+2$ dup & p.Ala108Glufs ${ }^{\star} 3$ \\
\hline 54 & $M$ & ND & ND & ND & ND & ND & ND \\
\hline 56 & $M$ & Exon 1 & c. $1 A>G$ & p.Met1? & Exon 4 & c. $352 \mathrm{G}>\mathrm{C}$ & p.Glu118GIn \\
\hline 57 & $\mathrm{~F}$ & Exon 1 & c. $1 A>G$ & p.Met1? & Exon 4 & c. $352 \mathrm{G}>\mathrm{C}$ & p.Glu118GIn \\
\hline 61 & $\mathrm{~F}$ & Exon 2 & c. $119 \mathrm{G}>\mathrm{C}$ & p.Arg40Pro & Exon 2 & c. $119 \mathrm{G}>\mathrm{C}$ & p.Arg40Pro \\
\hline 62 & $\mathrm{~F}$ & NF & NF & NF & $N F$ & NF & $\mathrm{NF}$ \\
\hline 63 & M & ND & ND & ND & ND & ND & ND \\
\hline 64 & M & ND & ND & ND & ND & ND & ND \\
\hline 67 & M & Intron 4 & c. $400+2$ dup & p.Ala108Glufs ${ }^{\star} 3$ & Exon 5 & c.521_523del & p.Phe174del \\
\hline
\end{tabular}

ND: not determined; NF: not found

One symbol for one family $(\sim, \bullet, \bullet)$

Table 3: APRT gene Mutations in adult patients (> 16 years).

to its rarity, wide spectrum of disease expression over the course of lifetime, from childhood to adulthood, and common symptoms among different disorders. Delay in diagnosis is common, with disease identification only after the onset of renal failure or when there is disease recurrence in the transplanted kidney after transplantation. Therefore, it is important that the diagnosis of APRT deficiency is considered in any patient with childhood onset kidney stones, frequent recurrences of urolithiasis in adolescence or adulthood, nephrocalcinosis or unexplained renal failure at any age as well as in patients with a family history of such disease manifestations.

Diagnosis relies on analysis of stone materials by infrared spectroscopy or detection of 2,8-DHA crystals in the urine which can be identified by polarized microscopy as typical 2,8-DHA crystals, round and reddish-brown with a characteristic central Maltese cross pattern (Figure 5). The identification of 2,8-DHA in urine and/or stone is pathognomonic of APRT deficiency but is confirmed by measurement of APRT activity in erythrocyte lysates and/or by molecular genetic testing.

\section{Crystalluria and stone analysis}

Crystalluria examination is performed by light microscopy using a polarizing microscope. Stone analysis combined morphologic examination by stereomicroscope and infrared spectroscopy as previously reported by Daudon et al. [43,44]. Crystals in renal biopsies are identified by polarizing microscopy from frozen biopsies or biopsies embedded in a paraffin matrix. Briefly, $5 \mu \mathrm{m}$ tissue slices are 


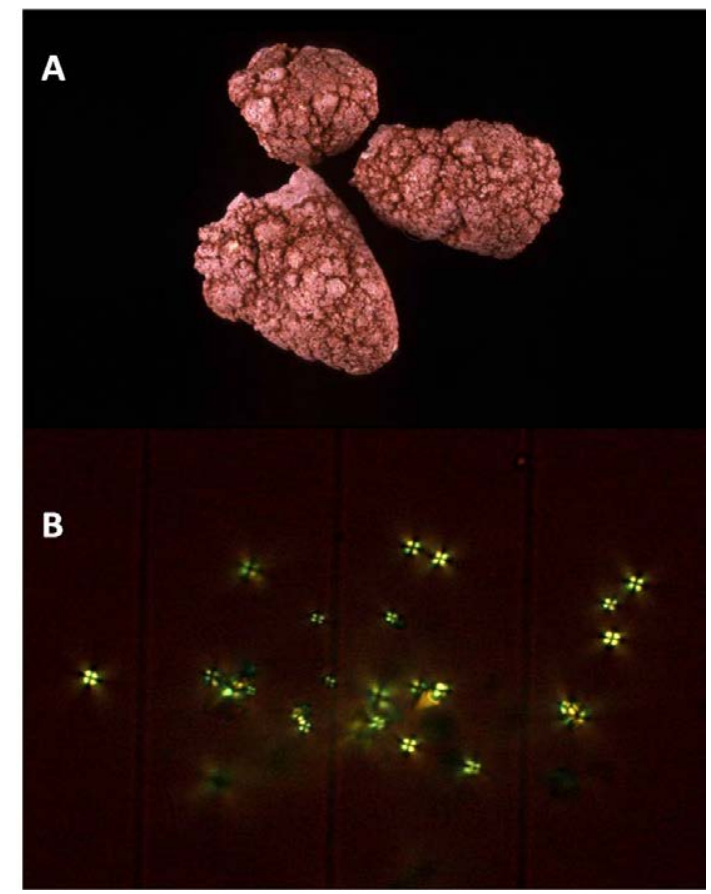

Figure 5: (A) Urinary calculi made of pure 2,8-dihydroxyadenine. (B) Typical spherical crystals of 2,8-dihydroxyadenine in urine. Crystals exhibit a central Maltese cross when viewed by polarized light microscopy.

spread out on a calcium fluoride plate or on a low-e microscope slide and directly examined using a Fourier transform infrared microscopy (FTIRM) $[45,46]$.

Renal biopsy is theoretically not required for diagnosis, given that 2,8-DHA crystals can be identified in urine and/or stone. However, some patients may also present with renal failure in the absence of stones; for these patients renal biopsy can demonstrate the presence of 2,8-DHA crystals into the renal parenchyma and lead to diagnosis of APRT deficiency. Renal biopsy typically shows severe tubular injury and precipitation of crystals within the tubular lumen and in renal interstitium. It must be emphasized that crystals in renal biopsy often lack the typical features of 2,8-DHA crystals that can be observed in urine. We recommend that crystals seen in renal biopsy specimen be fully characterized in order to avoid confusion with other crystalline deposits, especially uric acid and calcium oxalate. Whenever available, the combination of polarizing microscopy and FTIRM is a very reliable method for characterizing crystals in renal biopsy $[45,46]$.

Stone analysis should combine morphological examination under stereomicroscope and analysis using infrared spectroscopy or X-ray crystallography, which will unambiguously demonstrate the 2,8-DHA nature of the stone [43-45].

\section{APRT Activity in Erythrocyte Lysates}

Measurement of APRT enzyme activity in erythrocyte lysates is a useful tool for diagnosis of APRT deficiency because APRT activity assay demonstrates abnormal values in virtually all individuals with APRT deficiency at the homozygous or compound heterozygous state ( $0 \%$ in type I and less than $30 \%$ in type II). Heterozygotes carrying one $\mathrm{APRT}^{*} \mathrm{Q} 0$ and one normal allele show decreased but still detectable APRT activity (in our experience 10 to $55 \%$ of normal value).
Heterozygotes for APRT ${ }^{\star}$ J have more than $50 \%$ of the mean APRT activity of control subjects, which overlaps with normal levels $[47,48]$.

APRT enzyme activity is measured in erythrocytes lysates using radiolabeled 14C-adenine in a chromatographic assay, as previously described [49-51]. Unfortunately availability of APRT activity assay is restricted to a few laboratories in most countries.

\section{Molecular genetic testing}

Mutations in the aprt gene can be determined by sequencing of PCR-amplified DNA, which can be readily performed given the small size of APRT gene [1]. The diagnosis is confirmed if genetic test finds functionally significant mutations in both alleles (Tables 2 and 3 ).

\section{Other metabolic investigations}

Except for the excretion of adenine and its metabolites, no other biochemical abnormalities have been recorded. Developing an urinary 2,8-DHA assay for screening and for treatment monitoring is one of the objectives of the APRT Deficiency Research Program, which is part of the international Rare Kidney Stone Consortium (RKSC) (rarekidneystones.org) [12,52].

\section{Diagnostic Strategy}

Testing strategy may vary depending on the local availability of diagnostic tests. It may also depend on whether the aim is to establish diagnosis in a proband (individual without a family history of APRT deficiency) or to screen relatives of a patient with APRT deficiency.

\section{Strategy for diagnosis in a proband}

Screening for APRT deficiency in all cases of urolithiasis occurring in children, recurrent urolithiasis (especially if stones are radiolucent) and history of urolithiasis associated with acute or chronic kidney disease of uncertain cause, including ESRD patients and renal transplant recipients is recommended.

Diagnosis primarily relies on the recognition of 2,8-DHA in stones or crystals. Stone analysis should be performed whenever a stone is available, even if it was passed a long time ago. Urine microscopy examination should be systematically done. Infrared spectroscopy analysis of crystalluria is recommended when 2,8-DHA is suspected or, more generally, when crystals of uncertain nature are observed.

As discussed above, renal biopsy is theoretically not required for the diagnosis of 2,8-DHA nephropathy, given that 2,8-DHA crystals can be detected in urine in almost all affected individuals. Whenever histopathological findings consistent with crystalline nephropathy are observed, full characterization of the nature of crystals is mandatory. Such findings should prompt clinicians to search for crystals in urine. Crystals can be characterized in renal biopsy specimen using FTIRM. However, this technique is restricted to a few laboratories and crystalluria study is often an easier way to identify 2,8-DHA crystals.

Although the presence of 2,8-DHA in crystals or stones is pathognomonic of APRT deficiency, APRT activity measurement and/or genetic testing are recommended to confirm diagnosis. Measurement of APRT activity is particularly useful when no stone is available and crystalluria cannot be studied (e.g. anuric patient). We strongly recommend APRT activity measurement in ESRD patients awaiting renal transplantation who have a history of urolithiasis, unless the etiology of renal failure or the nature of stones has been clearly established.

Genetic testing is useful as a confirmatory test but is not intended 
as a primary screening procedure. Identifying the disease-causing mutations is of great relevance for familial screening once diagnosis has been confirmed in the proband.

\section{Strategy for familial screening}

Once APRT deficiency diagnosed in a patient and the mutations causing APRT deficiency have been identified in the proband, genetic testing screening of the kindred is recommended. Considering the autosomal recessive transmission, investigations should be focused on siblings. Genetic test should be used as first intention test. APRT activity measurement in erythrocytes may be also useful if causative mutations could not be found in the proband. We found that first degree relatives heterozygous for aprt mutation had residual APRT erythrocyte activity (10-55\% of normal value) and were completely asymptomatic (data not shown), as previously reported [1].

It is important to keep in mind that affected individuals may be asymptomatic but are still at risk to develop complications if the disease remains undiagnosed and untreated. All siblings, symptomatic or not, should therefore be investigated for APRT deficiency. Urine microscopy examination should be also performed. Considering the risk, although very small, of false negative, urine microscopy should not be used alone to screen at-risk relatives. Further investigations, including assessment of renal function and imaging studies, are warranted in individuals found with biallelic mutations, decreased APRT activity, or 2,8-DHA crystals in urine. As discussed above, 2,8DHA crystals are usually absent in the urine of heterozygotes.

\section{Treatment of APRT Deficiency}

Adequat treatment of APRT deficiency with allopurinol, which inhibits XDH thereby blocking the formation of 2,8-DHA from adenine (Figure 1), prevents kidney stone formation and the development of renal failure in most patients $[7,15,21]$. Allopurinol efficiently prevents the occurrence or progression of 2,8-DHA nephropathy, however, kidney disease can be irreversible at diagnosis, especially when tubulointerstitial lesions are advanced.

The usual dosage of allopurinol is $300-600 \mathrm{mg} / \mathrm{day}$ in adults and $5-10 \mathrm{mg} / \mathrm{kg} /$ day in children, which efficiently prevents 2,8-DHA crystal formation in most patients. In adults, we recommend initiating allopurinol therapy at a dose of $300 \mathrm{mg} / \mathrm{day}$, which is sufficient to achieve good control of the disease in most patients. Dosage should be subsequently increased in patients with persistent crystalluria and must be adapted when renal function is impaired. This treatment is welltolerated in most patients and children treated show normal growth and development [21]. Febuxostat, a more recent XDH inhibitor [53], may be an alternative for allopurinol intolerant patients and has also been used successfully [12,54]. In the absence of data available, no recommendation can be made regarding Febuxostat dosage in APRT deficiency.

All individuals with APRT deficiency, symptomatic or not, should receive life-long therapy with a $\mathrm{XDH}$ inhibitor. Patients and their families should be educated on the importance of life-long therapy and the risk of developing urolithiasis and 2,8-DHA nephropathy if the treatment is stopped. Treatment with allopurinol with appropriate dose adjustment is of paramount importance in patients undergoing renal transs antion in order to prevent recurrence of 2,8-DHA nephropathy, wm ch often leads to rapid and irreversible loss of allograft function $[1,8,9,10,26,27,55]$.

In dialysis patients awaiting a kidney transplant, it seems far preferable to initiate allopurinol therapy and achieve stable metabolic control prior to transplantation rather than initiation of $\mathrm{XDH}$ inhibition only after a new kidney has been implanted. This is especially true in view of limited understanding of the likelihood of systemic 2,8DHA accumulation in APRT-deficient patients with ESRD.

Along with $\mathrm{XDH}$-inhibiting drugs, patients should be advised to increase fluid intake to at least 2.5 liters of water per day (in adults) and to avoid purine rich food. Urinary alkalinization is not recommended, as 2,8-DHA remains very insoluble at any $\mathrm{pH}$ values [4].

Available data are limited regarding urological management of 2,8-DHA stones. In our experience, patients can benefit from various procedures, including extracorporeal shock-wave lithotripsy, endoscopy and surgery, as for the treatment of other types of stones.

The medical supervision of patients with APRT deficiency includes monitoring of renal function, quantitative analysis of 2,8DHA crystalluria, and renal ultrasound every 6 to 12 months in stable patients. Disappearance or at least a drastic reduction of the number of urinary 2,8-DHA crystals is usually observed under therapy and is considered an adequate treatment response [1,21]. A minority of patients experiences stone recurrence despite treatment [1,21]. Lack of adherence to treatment or insufficient dosing of allopurinol should be suspected in such situations or persistent crystalluria.

\section{Conclusion}

APRT deficiency is an under-recognized cause of kidney stones and crystalline nephropathy progressing over time to ESRD in a significant proportion of untreated individuals. In children, recurrent kidney stone disease and nephrocalcinosis, particularly in pre-pubertal children, should alert the physician to the possibility of an inborn error of purine metabolism as the underlying cause. Unfortunately, the lack of recognition and knowledge of APRT deficiency frequently resulted in a delay in diagnosis and treatment with $\mathrm{XDH}$ inhibitors sometimes with irreversible deterioration of renal function. A high index of suspicion coupled with early diagnosis may reduce or even prevent the serious long-term complications of this disease by using effective therapeutic intervention. The description of our series, the largest published to date, and the different diagnostic tools available will help clinicians in early detection of this under-recognized and treatable monogenic disease.

\section{Acknowledgements}

Authors report no conflict of interest relevant to this work.

$\mathrm{Dr} \mathrm{JL}$ Pérignon for his advice in the process of testing c.400+2dup in the newborn screening program and Dr N Dubois-Stringfellow for English improvement to the manuscript. We are very grateful for excellent assistance provided from all physicians who sent us clinical data and blood samples of patients: Pr Knebelman (Necker Hospital, Paris, France), Pr Bensman (Trousseau Hospital, Paris, France), Pr Deteix (Clermond Ferrand Hospital, France), Pr Jacquot (HEGP Universitary Hospital, Paris, France), Dr Snanoudj, Dr Heidet and Dr Guest (Necker Hospital Paris, France), Dr Llanas (Bordeaux Universitary Hospital, France), Dr Cassuto (Nice Hospital, France), Dr Bouvier (Draguignan, France), Dr Azema (Trousseau Hospital, Paris, France), Dr Demontis (Creil Hospital, France), Dr Kernaonet (Le Mans Hospital, France) Dr Le Monies de Sagazan (Roubaix Hospital, France), Dr Barrucand (Ugine, France), Dr Medeira (Santa Maria Hospital, Lisbon, Portugal), Dr Ferrando Monleon (La Ribeira Hospital, Valence, Spain), Dr Garnier and Dr Horen (Toulouse Universitary Hospital, France), Dr Elhani (Arinthod, France), Dr Boyer (Alpes du Sud Clinic, Gap, France), Dr Gie, Dr Gamber, Dr Taque (Rennes Universitary Hospital, France), Dr Vende (Bichat Universitary Hospital, Paris, France), Dr Rincé (Limoges Universitary Hospital, France), Dr Leonetti (St Brieuc Hospital, France), Dr Rechke (Melun Hospital, France), Dr Bigot (CHU Brest France), Dr Gaultier (CHICAS Hospital, Gap, France), Dr Veau (Lignière, France), Dr Palayret (Saint Calais, France), Dr Kolb (Ste Anne Clinic, Strasbourg, France) Dr Airoldi (Maggiore Hospital, Novara, Italy), Dr Heritier (CHR La Rochelle France) Dr Pelissier (Manosque, France), Dr Canavese (Maggiore Hospital, Novara, Italy) Dr Stratta (Maggiore Hospital, Novara, Italy), Dr Marra (Milano, Italy), Dr Glachant 
Citation: Picot IC, Ledroit M, Mockel L, Droin V, Daudon M, et al. (2014) Adenine Phosphoribosyltransferase Deficiency: An Under-Recognized Cause of Urolithiasis and Renal Failure. J Nephrol Ther 4: 173. doi:10.4172/2161-0959.1000173

Page 10 of 11

(Bourg en Bresse Hospital, France), Dr Gatault (Tours, France), Dr Maynard (Chambery Hospital, France), Dr Tostivint (CHU Pitié-Salpétrière, Paris, France), Pr Bardin (CHU Lariboisière, Paris, France), Pr Subra (CHU Angers, France), Dr Dheu (CHU Strasbourg, France), Dr Gartska and Dr Glowinski (CHU Lille, France, Dr Boussemart (Le Mans Hospital, France).

\section{References}

1. Bollée G, Dollinger C, Boutaud L, Guillemot D, Bensman A, et al. (2010) Phenotype and genotype characterization of adenine phosphoribosyltransferase deficiency. J Am Soc Nephrol 21: 679-688.

2. Bollée G, Harambat J, Bensman A, Knebelmann B, Daudon M, et al. (2012) Adenine phosphoribosyltransferase deficiency. Clin J Am Soc Nephrol 7: 15211527.

3. Ericson A, Groth T, Niklasson F, de Verdier CH (1980) Plasma concentration and renal excretion of adenine and 2,8-dihydroxyadenine after administration of adenine in man. Scand J Clin Lab Invest 40: 1-8.

4. Sahota A, Tischfield J, Kamatani N, Simmonds HA (2001) APRT deficiency and 2,8-DHA urolithiasis. In: Scriver CR, Beaudet AL, Sly WS, Valle D (eds) The Metabolic and Molecular Bases of Inherited Diseases. 8th ed. New York: McGraw-Hill, pp: 2571-2584.

5. Edvardsson V, Palsson R, Olafsson I, Hjaltadottir G, Laxdal T (2001) Clinical features and genotype of adenine phosphoribosyltransferase deficiency in iceland. Am J Kidney Dis 38: 473-480.

6. Nasr SH, Sethi S, Cornell LD, Milliner DS, Boelkins M, et al. (2010) Crystalline nephropathy due to 2,8-dihydroxyadeninuria: an under-recognized cause of irreversible renal failure. Nephrol Dial Transplant 25: 1909-1915.

7. Edvardsson VO, Goldfarb DS, Lieske JC, Beara-Lasic L, Anglani F, et al (2013) Hereditary causes of kidney stones and chronic kidney disease. Pediatr Nephrol 28: 1923-1942.

8. Eller P, Rosenkranz AR, Mark W, Theurl I, Laufer J, et al. (2004) Four consecutive renal transplantations in a patient with adenine phosphoribosyltransferase deficiency. Clin Nephrol 61: 217-221.

9. Stratta P, Fogazzi GB, Canavese C, Airoldi A, Fenoglio R, et al. (2010) Decreased kidney function and crystal deposition in the tubules after kidney transplant. Am J Kidney Dis 56: 585-590.

10. Kaartinen K, Hemmilä U, Salmela K, Räisänen-Sokolowski A, Kouri T, et al. (2014) Adenine phosphoribosyltransferase deficiency as a rare cause of renal allograft dysfunction. J Am Soc Nephrol 25: 671-674.

11. Doppler W, Hirsch-Kauffmann M, Schabel F, Schweiger M (1981) Characterization of the biochemical basis of a complete deficiency of the adenine phosphoribosyl transferase (APRT). Hum Genet 57: 404-410.

12. Edvardsson VO, Palsson R, SahotaA(2012)Adenine Phosphoribosyltransferase Deficiency. In: review G, ed. 2012/08/31 ed. Seattle: University of Washington.

13. Kamatani N, Terai C, Kuroshima S, Nishioka K, Mikanagi K (1987) Genetic and clinical studies on 19 families with adenine phosphoribosyltransferase deficiencies. Hum Genet 75: 163-168.

14. Broderick TP, Schaff DA, Bertino AM, Dush MK, Tischfield JA, et al. (1987) Comparative anatomy of the human APRT gene and enzyme: nucleotide sequence divergence and conservation of a nonrandom CpG dinucleotide arrangement. Proc Natl Acad Sci U S A 84: 3349-3353.

15. Chen J, Sahota A, Laxdal T, Scrine M, Bowman S, et al. (1991) Identification of a single missense mutation in the adenine phosphoribosyltransferase (APRT) gene from five Icelandic patients and a British patient. Am J Hum Genet 49: 1306-1311.

16. Deng L, Yang M, Frund S, Wessel T, De Abreu RA, et al. (2001) 2,8-Dihydroxyadenine urolithiasis in a patient with considerable residual adenine phosphoribosyltransferase activity in cell extracts but with mutations in both copies of APRT. Mol Genet Metab 72: 260-264.

17. Hidaka Y, Palella TD, O'Toole TE, Tarle SA, Kelley WN (1987). Human adenine phosphoribosyltransferase. Identification of allelic mutations at the nucleotide level as a cause of complete deficiency of the enzyme. J Clin Invest 80: 14091415.

18. Hidaka Y, Tarle SA, Fujimori S, Kamatani N, Kelley WN, et al. (1988) Human adenine phosphoribosyltransferase deficiency. Demonstration of a single mutant allele common to the Japanese. J Clin Invest 81: 945-50.

19. Kamatani N, Hakoda M, Otsuka S, Yoshikawa H, Kashiwazaki S (1992)
Only three mutations account for almost all defective alleles causing adenine phosphoribosyltransferase deficiency in Japanese patients. J Clin Invest 90 130-135.

20. Ceballos-Picot I, Perignon JL, Hamet M, Daudon M, Kamoun P (1992) 2,8-Dihydroxyadenine urolithiasis, an underdiagnosed disease. Lancet 339 1050-1051.

21. Harambat J, Bollée G, Daudon M, Ceballos-Picot I, Bensman A; APRT Study Group (2012) Adenine phosphoribosyltransferase deficiency in children. Pediatr Nephrol 27: 571-579.

22. Marra G, Vercelloni PG, Edefonti A, Manzoni G, Pavesi MA, et al. (2012) Adenine phosphoribosyltransferase deficiency: an underdiagnosed cause of lithiasis and renal failure. JIMD Rep 5: 45-48.

23. Debray H, Cartier P, Temstet A, Cendron J (1976) Child's urinary lithiasis revealing a complete deficit in adenine phosphoribosyl transferase. Pediat Res 10: 762-766.

24. Greenwood MC, Dillon MJ, Simmonds HA, Barratt TM, Pincott JR, et al. (1982) Renal failure due to 2,8-dihydroxyadenine urolithiasis. Eur J Pediatr 138: 346 349.

25. Yagisawa T, Yamazaki Y, Toma H, Kamatani N (1999) Radiopaque 2,8-dihydroxyadenine lithiasis. Int Urol Nephrol 31: 141-143.

26. Benedetto B, Madden R, Kurbanov A, Braden G, Freeman J, et al. (2001) Adenine phosphoribosyltransferase deficiency and renal allograft dysfunction. Am J Kidney Dis 37: E37.

27. Glicklich D, Gruber HE, Matas AJ, Tellis VA, Karwa G, et al. (1988) 2,8-dihydroxyadenine urolithiasis: report of a case first diagnosed after renal transplant. Q J Med 68: 785-793.

28. Vernon HJ, Osborne C, Tzortzaki EG, Yang M, Chen J, et al. (2005) Aprt/ Opn double knockout mice: osteopontin is a modifier of kidney stone disease severity. Kidney Int 68: 938-947.

29. Delbarre F, Auscher C, Amor B, de Gery A (1973) Gout with adenine phosphoribosyl transferase deficiency. Adv Exp Med Biol 41: 333-339.

30. Emmerson BT, Gordon RB, Thompson L (1975) Adenine phosphoribosyltransferase deficiency: its inheritance and occurrence in a female with gout and renal disease. Aust N Z J Med 5: 440-446.

31. Sahota A, Chen J, Behzadian MA, Ravindra R, Takeuchi H, et al. (1991) 2,8-Dihydroxyadenine lithiasis in a Japanese patient heterozygous at the adenine phosphoribosyltransferase locus. Am J Hum Genet 48: 983-989.

32. Ceballos-Picot I, Guest G, Moriniere V, Mockel L, Daudon M, et al. (2011) Maternal uniparental disomy of chromosome 16 in a patient with adenine phosphoribosyltransferase deficiency. Clin Genet 80: 199-201.

33. Chen J, Sahota A, Martin GF, Hakoda M, Kamatani N, et al. (1993) Analysis of germline and in vivo somatic mutations in the human adenine phosphoribosyltransferase gene: mutational hot spots at the intron 4 splice donor site and at codon 87. Mutat Res 287: 217-225

34. Sahota A, Chen J, Boyadjiev SA, Gault MH, Tischfield JA (1994) Missense mutation in the adenine phosphoribosyltransferase gene causing 2,8-dihydroxyadenine urolithiasis. Hum Mol Genet 3: 817-818.

35. Mimori A, Hidaka Y, Wu VC, Tarlé SA, Kamatani N, et al. (1991) A mutant allele common to the type I adenine phosphoribosyltransferase deficiency in Japanese subjects. Am J Hum Genet 48: 103-107.

36. Gathof BS, Sahota A, Gresser U, Chen J, Stambrook PS, et al. (1991) A splice mutation at the adenine phosphoribosyltransferase locus detected in a German family. Adv Exp Med Biol 309B: 83-86

37. Menardi C, Schneider R, Neuschmid-Kaspar F, Klocker H, Hirsch-Kauffmann $M$, et al. (1997) Human APRT deficiency: indication for multiple origins of the most common Caucasian mutation and detection of a novel type of mutation involving intrastrand-templated repair. Hum Mutat 10: 251-255.

38. Sahota A, Chen J, Stambrook PJ, Tischfield JA (1991) Mutational basis of adenine phosphoribosyltransferase deficiency. Adv Exp Med Biol 309B: 73-76.

39. Johnson LA, Gordon RB, Emmerson BT (1977) Adenine phosphoribosyltransferase: a simple spectrophotometric assay and the incidence of mutation in the normal population. Biochem Genet 15: 265-272.

40. Srivastava SK, Villacorte D, Beutler E (1972) Correlation between adenylate metabolizing enzymes and adenine nucleotide levels of erythrocytes during blood storage in various media. Transfusion 12: 190-197. 
Citation: Picot IC, Ledroit M, Mockel L, Droin V, Daudon M, et al. (2014) Adenine Phosphoribosyltransferase Deficiency: An Under-Recognized Cause of Urolithiasis and Renal Failure. J Nephrol Ther 4: 173. doi:10.4172/2161-0959.1000173

41. Simmonds H, Van Acker KJ, Sahota AS (1992) 2,8-Dihydroxyadenine urolithiasis. Lancet 339: 1295-1296.

42. Edvardsson VO, Palsson R, Sahota A (1993-2014) Adenine Phosphoribosyltransferase Deficiency. In: Pagon RA, Adam MP, Ardinger $\mathrm{HH}$, Bird TD, Dolan CR, Fong CT, Smith RJH, Stephens K, editors. GeneReviewsÂ $®$ [Internet]. Seattle (WA): University of Washington, Seattle.

43. Daudon M, Bader CA, Jungers $P$ (1993) Urinary calculi: review of classification methods and correlations with etiology. Scanning Microsc 7: 1081-1104.

44. Daudon M, Jungers $P(2004)$ Clinical value of crystalluria and quantitative morphoconstitutional analysis of urinary calculi. Nephron Physiol 98: p31-36.

45. Estepa-Maurice L, Hennequin C, Marfisi C, Bader C, Lacour B, et al. (1996) Fourier transform infrared microscopy identification of crystal deposits in tissues: clinical importance in various pathologies. Am J Clin Pathol; 105: 576582

46. Dessombz A, Bazin D, Dumas P, Sandt C, Sule-Suso J, et al. (2011) Shedding light on the chemical diversity of ectopic calcifications in kidney tissues: diagnostic and research aspects. PLoS One 6: e28007.

47. Kamatani N, Kuroshima S, Terai C, Kawai K, Mikanagi K, et al. (1987) Selection of human cells having two different types of mutations in individual cells (genetic/artificial mutants). Application to the diagnosis of the heterozygous state for a type of adenine phosphoribosyltransferase deficiency. Hum Gene 76: $148-152$

48. Fujimori S, Akaoka I, Sakamoto K, Yamanaka H, Nishioka K, et al. (1985)
Common characteristics of mutant adenine phosphoribosyltransferases from four separate Japanese families with 2,8-dihydroxyadenine urolithiasis associated with partial enzyme deficiencies. Hum Genet 71: 171-176.

49. Cartier P, Hamet M (1968) [Purine phosphoribosyltransferase activity of human erythrocytes. Technic of determination]. Clin Chim Acta 20: 205-214.

50. Cartier P, Hamet M, Hamburger J (1974) [A new metabolic disease: the complete deficit of adenine phosphoribosyltransferase and lithiasis of 2,8-dihydroxyadenine]. C R Acad Sci Hebd Seances Acad Sci D 279: 883-886.

51. Ceballos-Picot I, Mockel L, Potier MC, Dauphinot L, Shirley TL, et al. (2009) Hypoxanthine-guanine phosphoribosyl transferase regulates early developmental programming of dopamine neurons: implications for LeschNyhan disease pathogenesis. Hum Mol Genet 18: 2317-2327.

52. Monico CG, Milliner DS (2011) Genetic determinants of urolithiasis. Nat Rev Nephrol 8: 151-162.

53. Becker MA, Schumacher HR Jr, Wortmann RL, MacDonald PA, Eustace D, et al. (2005) Febuxostat compared with allopurinol in patients with hyperuricemia and gout. N Engl J Med 353: 2450-2461.

54. Hesse A, Tiselius H-G, Siener R, Hoppe B (2009) 2,8-dihydroxyadenine stones. In: urinary stones diagnosis, treatment and prevention of recurrence. 3rd Ed, Karger, pp142-151.

55. Gagné ER, Deland E, Daudon M, Noël LH, Nawar T (1994) Chronic renal failure secondary to 2,8-dihydroxyadenine deposition: the first report of recurrence in a kidney transplant. Am J Kidney Dis 24: 104-107. 